\title{
A BOUND ON DETERMINANTS
}

\section{WILLIAM M. FRANK}

The following result bounds the determinant of an arbitrary matrix by the determinant of a positive semi-definite matrix. It is well known that in the case of (psd) positive semidefinite matrices of order $n$, a geometrical bound $M^{n}$ can be found for the determinant which constitutes a considerable improvement over the general Hadamard bound $\sim n^{n / 2}$ for arbitrary matrices. The result is not practically applicable in all cases but is of particular value in studying the rate of convergence of Fredholm expansions for certain types of kernels. But for the broader class of matrices covered by this theorem, no improvement on classical estimates of special types of matrices is found.

THEOREM. If the $n \times n$ matrix $A$ is a linear combination of hermitian positive semidefinite ( $h p s d)$ matrices $A_{i}(i=1,2, \cdots, m)$, i.e.,

$$
A=\sum_{i=1}^{m} \mu_{i} A_{i}
$$

where the $\mu_{i}$ are complex constants, then

$$
|\operatorname{det} A| \leqq \operatorname{det}\left(\sum_{i=1}^{m}\left|\mu_{i}\right| A_{i}\right) \text {. }
$$

Proof. The proof is by induction on $m$. We first prove it for the case $m=2$. Without loss of generality we set $\mu_{1}=1, \mu_{2}=\mu$.

Let $U$ diagonalize $A_{1}$, i.e.,

$$
U A_{1} U^{-1}=D \text { (diagonal). }
$$

The matrix

$$
N=U A_{2} U^{-1}
$$

is still hpsd

$$
\operatorname{det} A=\operatorname{det}(D+\mu N) .
$$

$\operatorname{det}(D+\mu N)$ has the following expansion as a polynomial in $\mu$ :

$$
\operatorname{det}(D+\mu N)=\sum_{s=0} \mu^{\varepsilon} \sum_{\alpha \in S(n ; \varepsilon)} N_{\alpha} D_{\alpha}^{\prime},
$$

Received by the editors January 23, 1964. 
where $S(n ; s)$ is the set of unordered sequences of length $s$ from the integers $1,2, \cdots, n . N_{\alpha}$ is the principal minor of $N$ formed from the rows and columns numbered $\alpha_{1}, \alpha_{2}, \cdots, \alpha_{s}$, i.e.,

$$
N_{\alpha}=\left|\begin{array}{cccc}
N_{\alpha_{1} \alpha_{1}} & N_{\alpha_{1} \alpha_{2}} \cdots & N_{\alpha_{1} \alpha_{\varepsilon}} \\
N_{\alpha_{2} \alpha_{1}} & N_{\alpha_{2} \alpha_{2}} \cdots & N_{\alpha_{2} \alpha_{s}} \\
\vdots & \vdots & \vdots \\
N_{\alpha_{\varepsilon} \alpha_{1}} & N_{\alpha_{\varepsilon} \alpha_{2}} \cdots & N_{\alpha_{\varepsilon} \alpha_{\varepsilon}}
\end{array}\right|
$$

and is 1 for the case $s=0 . D_{\alpha}^{\prime}$ is the product of all the diagonal elements of $D$ excluding the elements $D_{\alpha_{1} \alpha_{1}}, D_{\alpha_{2} \alpha_{2}}, \cdots, D_{\alpha_{s} \alpha_{s}}$. By virtue of the positive semidefinite character of $D$ and $N$

$$
N_{\alpha} \geqq 0, \quad D_{\alpha}^{\prime} \geqq 0,
$$

for all $\alpha \in S(n ; s)$. From equations (5), (6),

$$
\begin{aligned}
|\operatorname{det} A| & =|\operatorname{det}(D+\mu N)| \leqq \sum_{s=0}^{n}|\mu| \cdot \sum_{\alpha \in S(n ; s)} N_{\alpha} D_{\alpha}^{\prime} \\
& =\operatorname{det}(D+|\mu| N)=\operatorname{det}\left[U\left(A_{1}+|\mu| A_{2}\right) U^{-1}\right] \\
& =\operatorname{det}\left(A_{1}+|\mu| A_{2}\right),
\end{aligned}
$$

which proves the theorem for the case $m=2$.

Assume the theorem to be true for all values of $m \leqq M$. Again take $\mu_{1}=1$, and consider

$$
\operatorname{det} A=\operatorname{det}\left(\sum_{j=1}^{M+1} \mu_{j} A_{j}\right)=\operatorname{det}\left(A+A^{\prime}\right),
$$

where

$$
A^{\prime}=\sum_{j=2}^{M+1} \mu_{j} A_{j} .
$$

Let $U$ be the matrix which diagonalizes $A_{1}$, and define

$$
\begin{gathered}
U A_{1} U^{-1} \equiv D, \quad U A_{j} U^{-1} \equiv N^{(j)}, \quad(j \geqq 2), \quad U A^{\prime} U^{-1} \equiv N \\
|\operatorname{det} A|=|\operatorname{det}(D+N)| \leqq \sum_{s=0}^{n} \sum_{\alpha \in S(n ; s)} D_{\alpha}^{\prime}\left|N_{\alpha}\right| .
\end{gathered}
$$

$N_{\alpha}$, a principal minor of the matrix $N$, is the determinant of a matrix $N_{(\alpha)}$ which is itself of the form

$$
N_{(\alpha)}=\sum_{j=2}^{M+1} \mu_{j} N_{(\alpha)}^{(j)},
$$


where $N_{(\alpha)}^{(j)}$ is the corresponding minor matrix of $N^{(j)}$. Since the $N^{(j)}$ are hpsd the induction hypothesis for the case $m=M$ can be applied to $N_{(\alpha)}$ of equation (7):

$$
\left|N_{\alpha}\right|=\left|\operatorname{det} N_{(\alpha)}\right| \leqq \operatorname{det}\left(\sum_{j=2}^{M+1}\left|\mu_{j}\right| N_{(\alpha)}^{(j)}\right)
$$

and

$$
\begin{aligned}
|\operatorname{det} A| & \leqq \sum_{s=0}^{n} \sum_{\alpha \in S(n ; s)} D_{\alpha}^{\prime} \operatorname{det}\left(\sum_{j=2}^{M+1}\left|\mu_{j}\right| N_{(\alpha)}^{(j)}\right) \\
& =\operatorname{det}\left(D+\sum_{j=1}^{M+1}\left|\mu_{j}\right| N^{(j)}\right)=\operatorname{det}\left(\sum_{j=1}^{M+1}\left|\mu_{j}\right| A_{j}\right),
\end{aligned}
$$

which proves the theorem for $m=M+1$, and, therefore, in general.

An arbitrary matrix can be expressed in the form of equation (1) with $m=4$ since the hermitian and $i$ times the antihermitian part of an arbitrary matrix can each be expressed as a difference of two hpsd matrices. The decomposition of a hermitian matrix $A$ in the particular form $A=A_{1}-A_{2}\left(A_{1}, A_{2}\right.$ hpsd) is, however, not unique. The inequality

$$
|\operatorname{det} A|<\operatorname{det}\left(A_{1}+A_{2}\right)
$$

becomes an equality when $A_{1}$ and $A_{2}$ commute. This corresponds to $A_{1}=U^{-1} D_{+} U, A_{2}=U^{-1} D_{-} U$, where $U A U^{-1}=D$ is diagonal and $D_{+}$, $-D_{-}$are, respectively, the non-negative and nonpositive diagonal parts of $D$.

That the bound in equation (17) is apt to be generous can be seen in the case where $A$ itself is positive semidefinite. ${ }^{1}$ Then, from wellknown properties of psd matrices,

$$
0 \leqq \operatorname{det} A=\operatorname{det}\left(A_{1}-A_{2}\right) \leqq \operatorname{det} A_{1}-\operatorname{det} A_{2},
$$

and the upper bound in equation (18) is bounded by

$$
\operatorname{det} A_{1}-\operatorname{det} A_{2} \leqq \operatorname{det} A_{1}+\operatorname{det} A_{2} \leqq \operatorname{det}\left(A_{1}+A_{2}\right),
$$

which is the bound of equation (17). If $A$ and $B$ are real symmetric matrices it is known that [1] $|\operatorname{det}(A+i B)|>\operatorname{det} A$. From the above theorem this is supplemented by

$$
|\operatorname{det}(A+i B)| \leqq \operatorname{det}(A+B)
$$

\footnotetext{
1 I am indebted to Dr. F. Metcalf of the Institute for Fluid Dynamics of the University of Maryland for this observation.
} 


\title{
REFERENCES
}

1. E. F. Beckenbach and R. Bellman, Inequalities, Chapter 2, Section 8, Springer, Berlin, 1961.

\section{U. S. Naval ORdnance Laboratory ${ }^{2}$}

2 The author is on leave of absence and is presently at the Weizmann Institute.

\section{NOTE ON DIFFERENTIAL OPERATORS WITH A PURELY CONTINUOUS SPECTRUM}

\author{
F. ODEH
}

In [1], Kreith gave an example of a Sturm-Liouville operator with positive coefficients,

$$
\begin{aligned}
L u & =-\frac{1}{r(x)} \frac{d}{d x}\left(p(x) \frac{d u}{d x}\right)+q(x) u, . \quad 0 \leqq x<\infty, \\
u(0) & =0
\end{aligned}
$$

which has a purely continuous spectrum. The novelty of the example lies in the relatively weak assumptions on the potential $q$. Thus, in the case $p=r=1$, one need not assume that $q$ is integrable at infinity - compare [2, Chapter 9, Problem 4]-but it is sufficient to assume $q$ to be monotonically decreasing. In this note a similar theorem is given which holds in any number of dimensions. The proof, which applies to Kreith's case also, shows that the nonexistence of eigenfunctions may be ascribed to two different reasons depending on the asymptotic behavior of $q(x)$. In one simple case it is due to the boundary condition while in the other, and more important, case it is a consequence of the behavior of $q$ at infinity. For simplicity the proof is restricted to the case of Schroedinger's equation in three dimensions, defined in the exterior $X$ of a closed smooth surface $\Gamma$. Hence, we consider the eigenvalue problem,

$$
L u=-\Delta u+q u=\lambda u,
$$

subject to the boundary conditions

Received by the editors December 18, 1963. 